\title{
SIX-MINUTE WALK TEST PERFORMANCE AND THEECHOCARDIOGRAM AS TOOLS FOR CLINICAL AND FUNCTIONAL ASSESMENT OF PATIENTS WITH SYSTEMIC SCLEROSIS
}

Luciana Campanatti Palhares ${ }^{1, *}$, Zoraida Sachetto ${ }^{1}$, Vinicius Velangieri Soubihe ${ }^{1}$, Marília Paula Souza dos Santos ${ }^{1}$, Daniela Camargo de Oliveira ${ }^{1}$, Juliana Zonzini Gaino ${ }^{1}$, Ana Paula Toledo Del Rio ${ }^{1}$

1. Universidade Estadual de Campinas, Campinas (SP), Brazil.

*Corresponding author: anapauladelrio@hotmail.com

\section{BACKGROUND}

Systemic sclerosis (SSc) is a rare, chronic autoimmune disease, characterized by immunological events, endothelial dysfunction, fibroblast disturbance and progressive cutaneous-visceral fibrosis including the heart. The aim of this study was to correlate transthoracic echocardiogram (TTE) and six-minute walk test (6MWT) parameters with anthropometric measurements, disease characteristics, immunological profile (IP) and lung function.

\section{MATERIALS AND METHODS}

24 patients diagnosed with SSc, followed up at Division of Rheumatology of a tertiary hospital, were evaluated. The mean age was 43.7 ( \pm 11.28 ) years and 20 patients (83.3\%) were female. Transthoracic echocardiogram exams were performed at the echocardiography service, pulmonary function at the pulmonology service, blood collection at the clinical analysis laboratory; 6MWT was performed in all patients. The statistical analysis was performed through Kruskal-Wallis test and Spearman correlation coefficient with a $5 \%$ level of significance.

\section{RESULTS}

The analysis presented moderate correlation coefficients of the following parameters: left ventricular ejection fraction (LVEF) with forced vital capacity (FVC) 0.43 ( $p<0.04)$; measurement of pulmonary artery systolic pressure (PASP) and velocity of tricuspid regurgitant jet (VRT) with age respectively $0.45(p<0.02)$ and $0.50(p<0.03)$; walked distance (WD) with PSAP -0.50 ( $p<0.01)$ and VRT -0.58 ( $p<0.01$ ); left ventricular wall thickness (LVWT) with body mass index (BMI) 0.50 ( $p<0.02$ ). When evaluating IP, patients with positive Anti-Scl 70 had 7\% lower LVEF $(p<0.03)$.

\section{CONCLUSIONS}

Older patients had increased PASP and VTR values. Those with the worst PASP and VTR values walked the shortest distances. Patients with better FVC results had higher LVEF. Overweight patients had increased LVWT. The 6MWT and TTE are useful tools in the assessment of SSc patients. 